\title{
Discerning novel splice junctions derived from RNA-seq alignment: a deep learning approach
}

\author{
Yi Zhang ${ }^{1 *}$, Xinan Liu', James MacLeod ${ }^{2}$ and Jinze Liu'
}

\begin{abstract}
Background: Exon splicing is a regulated cellular process in the transcription of protein-coding genes. Technological advancements and cost reductions in RNA sequencing have made quantitative and qualitative assessments of the transcriptome both possible and widely available. RNA-seq provides unprecedented resolution to identify gene structures and resolve the diversity of splicing variants. However, currently available ab initio aligners are vulnerable to spurious alignments due to random sequence matches and sample-reference genome discordance. As a consequence, a significant set of false positive exon junction predictions would be introduced, which will further confuse downstream analyses of splice variant discovery and abundance estimation.

Results: In this work, we present a deep learning based splice junction sequence classifier, named DeepSplice, which employs convolutional neural networks to classify candidate splice junctions. We show (I) DeepSplice outperforms state-of-the-art methods for splice site classification when applied to the popular benchmark dataset HS3D, (II) DeepSplice shows high accuracy for splice junction classification with GENCODE annotation, and (III) the application of DeepSplice to classify putative splice junctions generated by Rail-RNA alignment of 21,504 human RNA-seq data significantly reduces 43 million candidates into around 3 million highly confident novel splice junctions.

Conclusions: A model inferred from the sequences of annotated exon junctions that can then classify splice junctions derived from primary RNA-seq data has been implemented. The performance of the model was evaluated and compared through comprehensive benchmarking and testing, indicating a reliable performance and gross usability for classifying novel splice junctions derived from RNA-seq alignment.
\end{abstract}

Keywords: Deep learning, Splice junction, RNA-seq, Exon splicing

\section{Background}

Technological improvements, reduced cost, and accessibility of RNA sequencing technologies have provided unprecedented visibility of the transcriptome through the deep sequencing of all mRNA transcripts present in a sample. Through analyses of mRNA-seq data, researchers now believe that $92-94 \%$ of mammalian protein-coding genes undergo alternative splicing, with roughly $86 \%$ of these containing a minor transcript isoform frequency of at least $15 \%$ in certain cell types, developmental time points, physiological states, or other

\footnotetext{
* Correspondence: yi.zhang@uky.edu

${ }^{1}$ Department of Computer Science, University of Kentucky, Lexington, KY 40506, USA

Full list of author information is available at the end of the article
}

conditions [1]. This is an $87-89 \%$ increase from 40 years ago when alternative exon structures from a single gene locus were first introduced and it was believed that only around $5 \%$ of genes in higher eukaryotes undergo alternative splicing [2].

The approach to defining exon junctions from RNA-seq data utilizes the subset of reads that have a gapped alignment to the reference genome. These reads can be aligned to two or more exons, indicating that there exist junctions joining adjacent exons. Whereas some mapping strategies [3-6] require pre-defined structural annotation of exon coordinates, more recently developed algorithms [7-11] can conduct ab initio alignment, which means that they do not rely on the existence of predetermined gene structure annotation and 
can potentially identify novel splice junctions between exons by the evidence of spliced alignments.

The accurate prediction of exon junctions is essential for defining gene structures and mRNA transcript variants. Splicing must be absolutely precise because the deletion or addition of even a single nucleotide at the splice junction would throw the subsequent three-base codon translation of the RNA out of frame [12]. However, novel splice junctions predicted by read alignments are not totally reliable, since the possibility of randomly mapping a short read up to 150 bases to the large reference genome is high [13], especially when gapped alignments with short anchoring sequences are permitted. In a recent report by Nellore et al. [14] that investigated splicing variation, 21,504 RNA-seq samples from the Sequenced Read Archive (SRA) were aligned to the human hg19 reference genome with Rail-RNA [15], identifying 42 million putative splice junctions in total. This value is 125 times the number of total annotated splice junctions in humans, making it impossible to admit that all of them actually exist. False positive splice junctions may lead to false edges in splice graphs, significantly increasing the complexity of the graphical structures [16]. Consequentially, this will impact the accuracy of splice variant inference algorithms as they often start from splice graphs derived from RNA-seq alignment [17].

Conventional strategies designed to filter out false positive exon splice junctions depend primarily on two properties: (1) the number and the diversity of reads mapped to the given splice junction [13]; and/or (2) the number of independent samples in which the specific exon splice junction is identified $[13,18]$. In general, higher read support and sample reoccurrence rate both enlarge the likelihood of being a true splice junction. These criteria have a positive correlation with the number of read alignments, which are dependent on the sampling depth of the particular sample. Exact thresholds are difficult to set due to varying sampling depth across samples. Additionally, due to both sequencing and alignment errors, a splice junction with both high read support and high sample reoccurrence may still be the result of systematic bias. In contrast, a splice junction that exists in a transcript with relatively low expression may still be functionally important [19]. Thus, further classification of putative splice junctions revealed by RNA-seq data is still necessary but remains a challenging issue.

Since the 1980s, a number of bioinformatic approaches have been developed for splice site prediction. Neural networks [20-22], support vector machines [2325], hidden Markov model [26-28], deep Boltzmann machines [29] and discriminant analysis [30, 31] have been applied to recognize splice sites in the reference genome of many given species. Neural networks, support vector machines and deep Boltzmann machines learn the complex features of neighborhoods surrounding the consensus dinucleotide AG/GT by a non-linear transformation. Hidden Markov models estimate position specific probabilities of splice sites by computing the likelihoods of candidate signal sequences. The discriminant analysis uses several statistical measures to evaluate the presence of specific nucleotides, recognizing splice sites without explicitly determining the probability distributions [28]. However, all these work treat donor and acceptor sites as independent events, failing to leverage the inherent relationships between the donor and acceptor during splicing.

In this paper, we develop a deep neural network-based approach to the classification of potential splice junctions. Our method is applicable to both splice site prediction and splice junction classification. First, instead of treating donor or acceptor splice sites individually, our method models the donor and acceptor splice sites as a functional pair. Thus, it is capable of capturing the remote relationships between features in both donor and acceptor sites that determine the splicing. Additionally, flanking subsequences from both exonic and intronic sides of the donor and acceptor splice sites will be used for learning and prediction, making it possible to understand the contribution of both coding and non-coding genomic sequences to the splicing. Our approach does not rely on sequencing read support or frequency of occurrence derived from experimental RNA-seq data sets, thus can be applied as an independent evidence for splice junction validation. Our experiments demonstrate that DeepSplice outperforms other state-of-the-art approaches [28, 32-36] when tested against a benchmarking dataset, Homo sapiens Splice Sites Database (HS3D), using a variety of evaluation metrics. Trained on an older version of the GENCODE project gene annotation data [37], we show that our algorithm can predict the newly annotated splice junctions with high accuracy and performs better than splice site-based approach. The application of DeepSplice to further classify putative intropolis human splice junction data by Nellore et al. [14] is able to eliminate around $83 \%$ unannotated splice junctions. We discover that the combinational information from the functional pairing of donor and acceptor sites facilitates the recognition of splice junctions and demonstrate from large amounts of sequencing data that non-coding genomic sequences contribute much more than coding sequences to the location of splice junctions [25, 38].

\section{Results}

We first applied our approach to a benchmark dataset HS3D [39] and compared the performance with other state-of-the-art approaches for donor and acceptor splice site classification. We then evaluated DeepSplice's performance by classifying annotated splice junctions from 
GENCODE gene annotation data [37]. Deep Taylor decomposition [40] was then applied for further interpretation of base level contribution of flanking splice sequence. Finally, we applied DeepSplice to intropolis [14], a newly published splice junction database with $42,882,032$ splice junctions derived from 21,504 samples. The detailed results are described below. Supplementary results are available in Additional file 1.

\section{DeepSplice outperforms state-of-the-art splice site prediction method}

We utilized HS3D [39] (Homo sapiens Splice Sites Data set, http://www.sci.unisannio.it/docenti/rampone/), a popular benchmark for measuring the quality of splice site classification methods. HS3D includes introns, exons and splice site sequences extracted from GeneBank Rel. 123. The splice site sequences in HS3D are with the length of 140 nucleotides. There are 2796 (2880) true donor (acceptor) splice sites and 271,937 $(329,374)$ false donor (acceptor) splice sites which all contain conserved GT (AG) dinucleotides. We constructed the 1:10 data set, which contains all the true splice sites and 27,960 (28,800) randomly selected false donor (acceptor) splice sites. Binary classifications were conducted to identify the actual splice sites on donor and acceptor splice site data separately.

DeepSplice was trained on donor and acceptor splice site sequences separately in order to compare with state-of-the-art approaches of splice site classification. The exact same number of training and testing splice site sequences from HS3D were used for all approaches. Table 1 summarizes the classification accuracies on the 1:10 data set by 10 -fold cross-validation. To measure the quality of the classification results, we employed sensitivity, specificity, and $Q^{9}$ which is the global accuracy measure calculated from both sensitivity and specificity scores. Since the published splice site classification methods do not provide public tools for training and testing, the results of SVM + B [32], MM1-SVM [28], DM-SVM [33], MEM [34] and LVMM2 [35] were obtained from [33, 35]. As shown in Table 1, DeepSplice outperforms other methods in both sensitivity and specificity for both donor and acceptor splice site classification. For donor splice sites, there is a 95\% likelihood that the confidence interval [0.0581, 0.0633] covers the true classification error of DeepSplice on the testing data. For acceptor splice sites, there is a 95\% likelihood that the confidence interval [0.0814, 0.0872] covers the true classification error of DeepSplice on the testing data.

To deduce the most suitable architecture for learning the patterns in splice site/junction sequences, we then compared DeepSplice against two other prominent types of neural networks, multilayer perceptron network and long short-term memory network, in terms of classifying HS3D data set by 10 -fold cross-validation. As shown in Fig. 1, DeepSplice with convolutional neural network exceeds the other architectures, achieving an auROC score of $0.983(0.974)$ on donor (acceptor) splice site classification and an auPRC score of $0.863(0.800)$ on donor (acceptor) splice site classification. LSTM achieved an auROC score of 0.960 (0.942) on donor (acceptor) splice site classification and an auPC score of $0.803(0.721)$ on donor (acceptor) splice site classification. MLP achieved an auROC score of 0.931 (0.914) on donor (acceptor) splice site classification and an auPRC score of 0.650 (0.559) on donor (acceptor) splice site classification. In general, convolutional neural network is a well-studied architecture, which outperforms other deep learning architectures in almost all kinds of applications currently [41]. Even for speech recognition, convolutional neural networks recently beat recurrent neural networks. In our application, convolutional layers efficiently learned the complex information of nucleotide neighborhoods.

\section{DeepSplice predicts newly annotated splice junctions with high accuracy}

Next, we evaluated the accuracy of DeepSplice in terms of splice junction classification. To achieve this, we trained DeepSplice using splice junctions extracted from the GENCODE annotation version 3c, and then tested the model on newly annotated splice junctions in the GENCODE annotation version 19. All GENCODE splice

Table 1 Evaluation of DeepSplice and state-of-the-art approaches for donor (acceptor) site classification on HS3D data set

\begin{tabular}{|c|c|c|c|c|c|c|}
\hline & \multicolumn{3}{|l|}{ Donor } & \multicolumn{3}{|l|}{ Acceptor } \\
\hline & Sensitivity & Specificity & $\mathrm{Q}^{9}$ & Sensitivity & Specificity & $Q^{9}$ \\
\hline LS-GKM & 0.8679 & 0.8516 & 0.8595 & 0.8403 & 0.8319 & 0.8361 \\
\hline$S V M+B$ & 0.9406 & 0.9067 & 0.9212 & 0.9066 & 0.8797 & 0.8920 \\
\hline MM1-SVM & 0.9256 & 0.9244 & 0.9247 & 0.8993 & 0.8869 & 0.8926 \\
\hline DM-SVM & 0.9469 & 0.9339 & 0.9399 & 0.9215 & 0.9073 & 0.9136 \\
\hline MEM & 0.9324 & 0.9275 & 0.9295 & 0.9153 & 0.8843 & 0.8978 \\
\hline LVMM2 & 0.9424 & 0.9242 & 0.9323 & 0.9122 & 0.8970 & 0.9039 \\
\hline DeepSplice & 0.9571 & 0.9376 & 0.9465 & 0.9337 & 0.9139 & 0.9232 \\
\hline
\end{tabular}


(a)

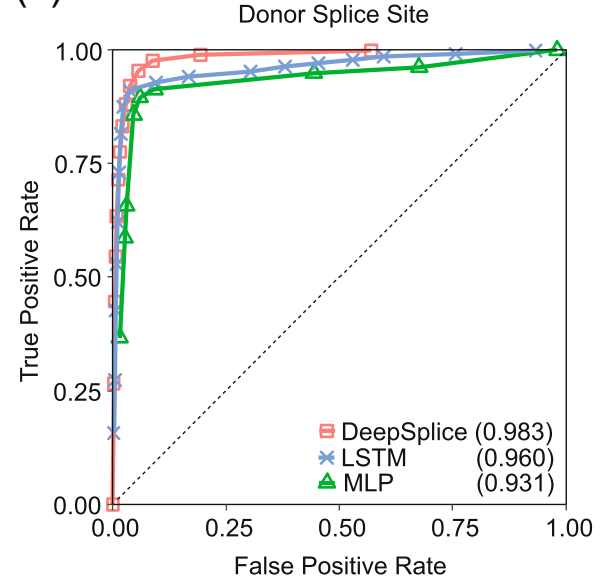

(b)

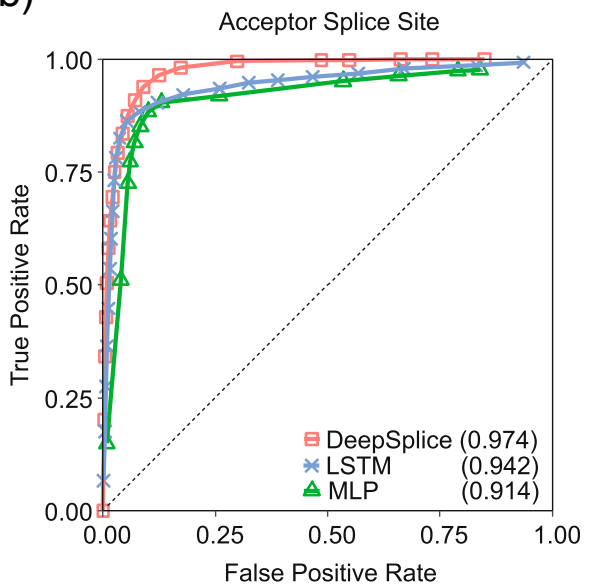

Fig. 1 The ROC curves of DeepSplice, multilayer perceptron network (MLP) and long short-term memory network (LSTM) for (a) donor splice site and (b) acceptor splice site classification on the HS3D data set by 10-fold cross-validation. DeepSplice with convolutional neural network exceeds the other deep learning architectures, achieving an auROC score of 0.983 (0.974) on donor (acceptor) splice site classification

junctions used for training and testing are experimental validated by RT-PCR amplification. The training set contains 521,512 splice junctions, and the testing set contains 106,786 splice junctions. In both training and testing sets, half of the splice junctions are annotated, and the rest are false splice junctions randomly sampled [42] from human reference genome (GRCh37/hg19).

We trained the first model by feeding the 521,512 training splice junction sequences to DeepSplice for a binary classification, splice junctions or not. In the meantime, we trained two other models separately by feeding the donor (acceptor) splice site sequences extracted from the 521,512 training splice junction sequences to DeepSplice for a binary classification, donor (acceptor) splice sites or not. This experiment was designed to determine whether making use of paired combinational information of donor and acceptor splice sites from a splice junction, instead of classifying donor or acceptor splice site individually, would ameliorate the quality of splice junction classification. In the first mode (Splice Junction Mode), the input splice junction sequences were with the length of 120 nucleotides, reflecting 30 nucleotides of upstream and downstream nucleotides for both donor and acceptor splice site. In the second mode (Donor+Acceptor Site Mode), the input splice junction sequences were split into two substrings with the length of 60 nucleotides and then fed to donor (acceptor) splice site classification model separately. For the second mode, we defined that the probability of a splice junction being classified as positive is the product of the probability of its donor splice site being classified as positive and the probability of its acceptor splice site being classified as positive, considering the two splice site classification events are statistically independent [43]. Figure 2 shows the ROC curves of the two modes. Splice Junction Mode achieved an auPRC score of 0.990, 0.987 for Donor+Acceptor Site Mode. Table 2 summarizes sensitivity, specificity, accuracy, and F1 score on the 106,786 testing splice junction sequences. Donor+Acceptor Site Mode acquires a higher specificity; however, Splice Junction Mode significantly outperforms Donor+Acceptor Site Mode in terms of sensitivity, accuracy, F1 score, auROC score, and auPRC score with substantially higher scores. In total, Splice

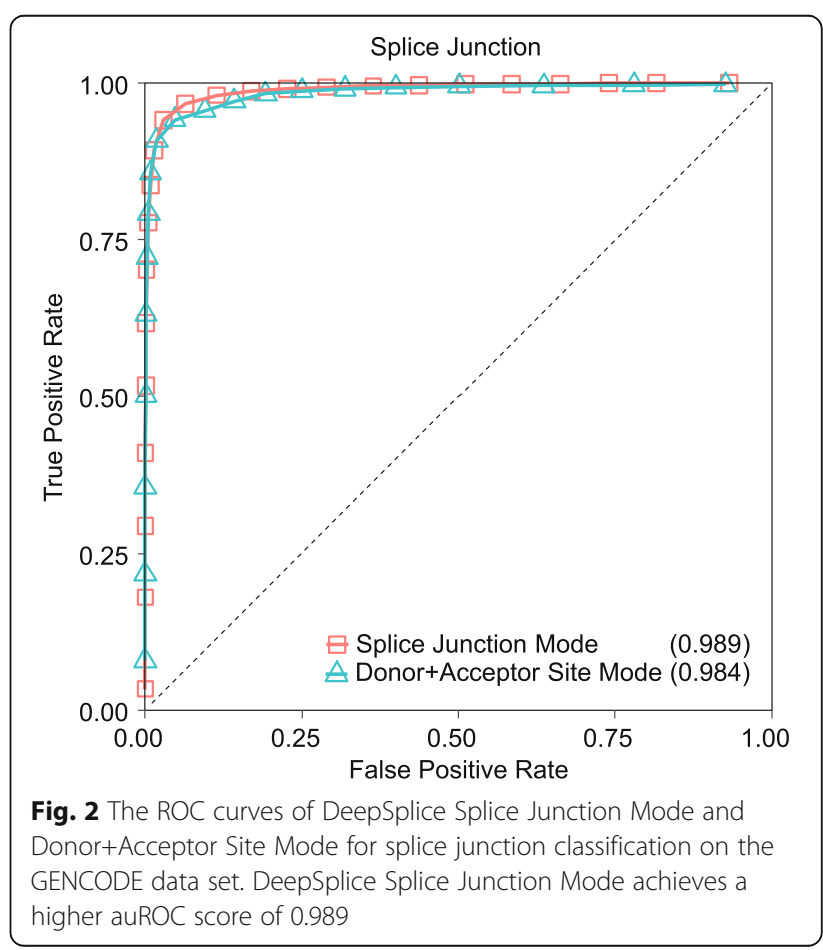


Table 2 Classification performance evaluation of different DeepSplice modes on GENCODE data set

\begin{tabular}{|c|c|c|c|c|c|}
\hline & & Sensitivity & Specificity & Accuracy & F1 score \\
\hline \multirow[t]{2}{*}{ Splice site classification } & Donor & 0.917 & 0.897 & 0.907 & 0.908 \\
\hline & Acceptor & 0.873 & 0.913 & 0.893 & 0.891 \\
\hline \multirow[t]{2}{*}{ Splice junction classification } & Splice Junction Mode & 0.943 & 0.968 & 0.956 & 0.955 \\
\hline & Donor + Acceptor Site Mode & 0.732 & 0.997 & 0.864 & 0.844 \\
\hline
\end{tabular}

Junction Mode predicted 50,340 out of 53,393 newly annotated splice junctions, which covered 9806 genes, 98.01\% of all newly annotated genes. Donor+Acceptor Site Mode detected 39,067 splice junctions from 9185 genes. There is a 95\% likelihood that the confidence interval [0.0432, 0.0456] covers the true classification error of DeepSplice on the testing splice junctions. These results indicate that the proposal splice junction classification in DeepSplice achieves high accuracy in identifying novel splice junctions in large data sets than conventional splice site classification.

\section{Interpretation of sequence features captured by DeepSplice}

There are highly conserved segments on splice junctions between exons and introns which help in the prediction of splice junctions by computational methods and decipher biological signals of splice junctions. We next further interpret which nucleotides contribute to the splicing process. This is achieved by the quantification of the contribution of nucleotides in splice junction sequences to the classification process using deep Taylor decomposition [40].

DeepSplice employs convolutional neural network with two convolutional layers. In the convolutional layer, we defined filters with a shape of $3 \times 1$, which means filters scan the input sequence with a window size of 3 to learn the information of nucleotide neighborhoods. DeepSplice fundamentally is not using a single base but rather 3-mers or subsequences of length 3 as its features. Then deep Taylor decomposition runs a backward pass on the convolutional neural network to sign contributions. The contribution score of each single base in DeepSplice reflects the aggregated importance of the three 3-mers it belongs to. We first used deep Taylor decomposition to decompose cross-validation results of the HS3D dataset in terms of input splice site sequences. For nucleotides in the testing splice site sequences, scores were assigned to present their contribution. We obtained a graphical representation from which it is possible to judge which region in the splice site sequences is of importance. Figure 3 shows the contribution of nucleotides to the final decision function of DeepSplice. In general, intron sequences carry more discriminative information than exon sequences in this analysis. We then applied deep Taylor decomposition to the results of splice junction classification with the GENCODE data set. Figure 4 shows the contribution distribution of nucleotides in the testing splice junction sequences. Regions of increased importance in splice junction classification are consistent with the result from splice site classification.

\section{DeepSplice classification of intropolis}

The intropolis v1 database [14] contains a large number of putative junctions found across 21,504 human RNA-seq samples in the Sequence Read Archive (SRA) from spliced read alignments to hg19 with Rail-RNA [15]. There are $42,882,032$ putative splice junctions in total, including $18,856,578$ canonical splice junctions containing flanking string GT-AG, 24,025,454 semi-canonical splice junctions containing flanking string AT-AC or GC-AG [44], and no non-canonical splice junctions which are not allowed by Rail-RNA. Table 3 lists the number of splice junctions in each category separated by the number of reoccurrence in samples and total read support across all samples in four scales: (a) equal to $1\{1\}$, (b) more than 1 and no greater than $10(1,10]$, (c) more than 10 and no greater than 1000 $(10,1000]$ and $(d)$ more than $1000(1000,+\infty)$. As listed in Table 3, for our analysis, we only retain splice junctions in intropolis that are supported by more than one sample, followed by the filtering of false splice junction sequences due to repetitive sequences. After this pre-processing, $5,277,046$ splice junctions were left for further classification.

The DeepSplice model was trained on 812,967 splice junctions including (1) 291,030 annotated splice junctions from GENCODE annotation version 19, (2) 271,937 false splice junctions generated from the HS3D data set, and (3) 250,000 randomly selected semi-canonical splice junctions with only one read support from intropolis. Overall, DeepSplice classified 3,063,698 splice junctions as positive. Figure 5(a) lists the proportions of positive canonical splice junctions, positive semi-canonical splice junctions and negatives from the classification results at different levels of average read support per sample. Splice junctions with average read support per sample more than 15 achieve a positive rate around $88 \%$. In contrast, for splice junctions with average read support per sample no more than 1, only $36 \%$ are identified as positives. There is a significant rise in the probability to obtain a positive splice junction with the increase of the average read support per sample. Around 99\% positive splice junctions 


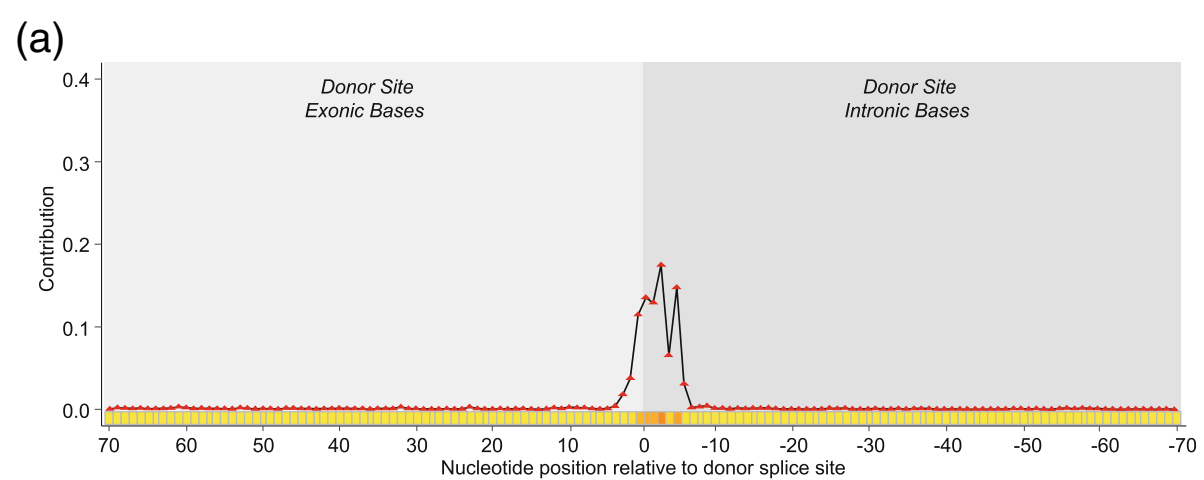

(b)

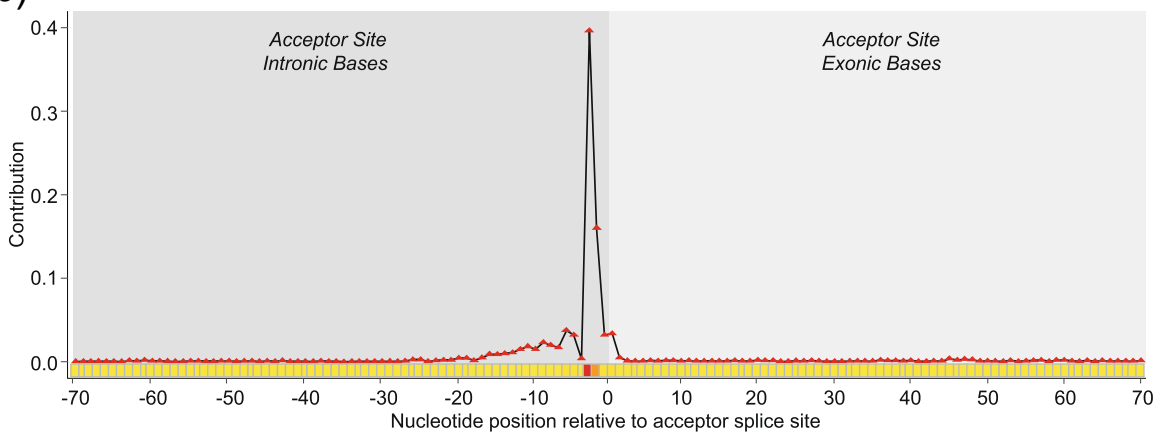

Fig. 3 Visualization of the contribution of nucleotides in the flanking splice sequences to the final decision function of DeepSplice on the HS3D dataset for (a) donor splice site and (b) acceptor splice site classification. For both donor and acceptor site classifiers, intronic bases close to GT-AG dinucleotides achieve the most importance in the classifiers. In general, intron sequences carry more discriminative information than exon sequences

contain the canonical flanking string. Figure 5(b) illustrates the proportions of positive semi-canonical and canonical splice junctions cumulatively with the increase of the average read support per sample.

To further clarify characteristics of the positives, we categorized splice junctions in intropolis based on annotated splice sites in GENCODE annotation: (1) splice junctions with both splice sites annotated, (2) splice junctions with the donor splice site annotated, (3) splice junctions with the acceptor splice site annotated, and (4) splice junctions with neither the donor nor acceptor splice sites annotated. Figure 6(a) shows the discrete proportions of negatives and positive splice junctions in each category above, given the average read support per sample. Results indicate that $97 \%$ of splice junctions with both sites annotated are classified as positives, while only $39 \%$ with both sites being novel are positive. Splice junctions connecting annotated splice sites also tend to be associated with higher read coverage. Figure 6(b) illustrates the proportions of positive splice junctions in each category cumulatively with the increase of the average read support per sample. Figure 7 shows positive splice junctions in intropolis near known protein-coding junctions show a periodic pattern, such that splice sites which maintain the coding frame of the exon are observed more often than those which disrupt frame. This observation recapitulates patterns seen in studies of noisy splicing [19].

\section{Discussion}

Even though splice junctions with high read support and/or high reoccurrence are more likely to be classified as real, a significant portion of relatively low-expressed splice junctions also carry true splicing signals. DeepSplice does not rely on sequencing read support, frequency of occurrence, or sequencing read length derived from experimental RNA-seq data sets, thus can be applied as an independent evidence for splice junction validation. The accumulation of RNA-seq data especially in different cell types, tissues and disease conditions will further consolidate the cell type-specificity and tissue-specificity of some of these junctions and their corresponding isoforms. DeepSplice may provide the first round of filtering of RNA-seq derived splice junctions for further structural validation, and studies that assess functional annotation of these splice junctions are warranted. DeepSplice could also extend its functionality to discriminate splice junctions that are highly or lowly supported by gene expression evidence and try to figure out what sequence patterns associate to this difference in future. For each input candidate splice junction, DeepSplice outputs a probability of being true, and the probability can be used as an 


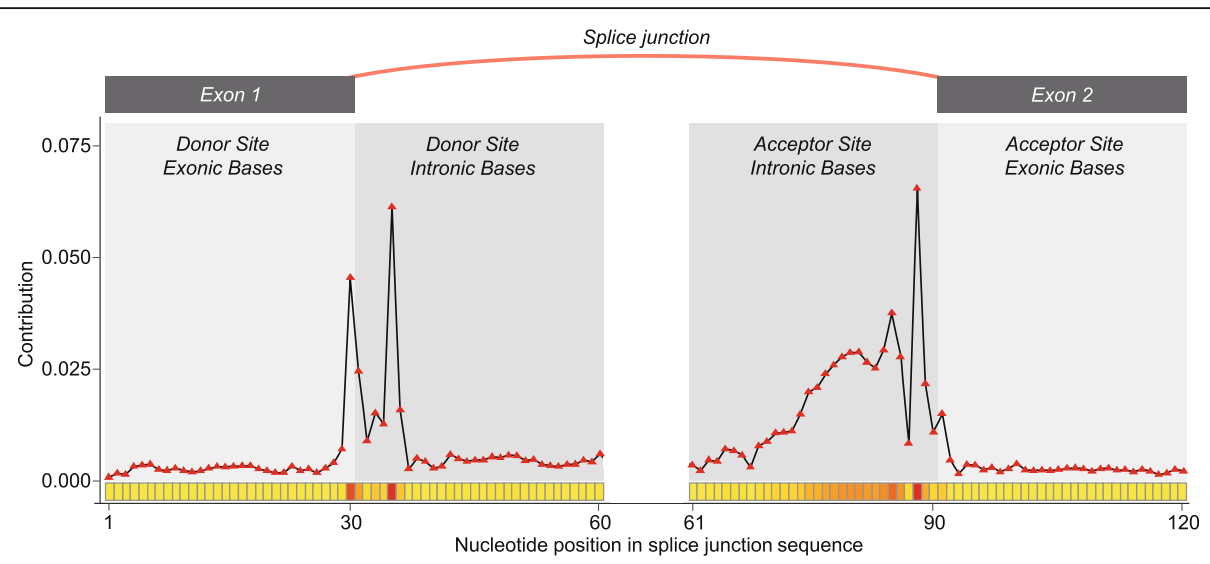

Fig. 4 Visualization of the contribution of nucleotides in the flanking splice sequences to the final decision function of DeepSplice on the GENCODE dataset for splice junction classification. The nucleotides in the proximity of a splice junction have the highest impact on the classification outcome. As observed in the splice site classifiers, the contribution distribution of nucleotides in the flanking splice sequences indicates that intron nucleotides carry more discriminative information than exon nucleotides

input feature to the studies for learning the tissue-regulated splicing code [45] and the splicing in human tissues with a wide range of known diseases [46].

It is also well known that splicing can be changed due to mutations around the splice sites. Future studies that use subject-specific genomic sequences instead of reference genome sequences may further improve the accuracy of the DeepSplice model and classification performance. Additionally, DeepSplice can be further extended to the prediction of non-canonical splicing [47] that existing annotation has not captured, including not only exonic but also splicing involving Alu elements, small exons, and recursive splicing. Besides the classification of linear junctions, the identification of non-linear splice junctions, such as circRNA junctions will also expand the functionality of DeepSplice.

\section{Conclusions}

Employing deep convolutional neural network, we develop DeepSplice, a model inferred from the sequences of annotated exon junctions that can then classify splice junctions derived from primary RNA-seq data, which can be applied to all species with sufficient transcript

Table 3 Distribution of splice junctions from intropolis given the reoccurrence in samples and total read support

\begin{tabular}{llllll}
\hline \multirow{2}{*}{ Splice junction number } & \multicolumn{4}{l}{ Reoccurrence in samples } \\
\cline { 3 - 6 } & $\{1\}$ & $(1,10]$ & $(10,1000]$ & $(1000,+\infty)$ \\
\hline Total reads & $\{1\}$ & $23 \mathrm{M}$ & - & - & - \\
& $(1,10]$ & $3331 \mathrm{~K}$ & $11 \mathrm{M}$ & - & - \\
& $(10,1000]$ & $91 \mathrm{~K}$ & $936 \mathrm{~K}$ & $3301 \mathrm{~K}$ & - \\
& $(1000,+\infty)$ & 38 & 187 & $124 \mathrm{~K}$ & $305 \mathrm{~K}$ \\
\hline
\end{tabular}

" $\mathrm{M}$ " stands for "million"

"K" stands for "thousand" annotation to use as training data. Results demonstrate that DeepSplice outperforms the state-of-the-art splice site classification tools in terms of both classification accuracy and computational efficiency. Our findings further indicate that valuable information is present in the nucleotide sequence local to the splice junction, data that conventional splice site prediction techniques discard. Nucleotide representations learned from the input sequences are meaningful and improve accuracy. The major application of DeepSplice is the classification of splice junctions rather than individual donor or acceptor sites. For learning on large datasets of putative splice junctions, DeepSplice is orders of magnitude faster than the best performing existing alternatives, which becomes increasingly common considering the tremendous amount of new RNA-seq data being generated.

\section{Methods}

DeepSplice employs a convolutional neural network (CNN, or ConvNet) to understand sequence features that characterize real splice junctions. The overall architecture of DeepSplice is shown in Fig. 8. In the supervised training step, $\mathrm{CNN}$ learns features that help to differentiate actual splice junctions from fake ones. In the inference step, the trained model uses the genomic sequence of the candidate splice junction and predicts the probability of it being a real splice junction. Deep Taylor decomposition [40] of the CNN is used to explain to what extent each nucleotide in the candidate splice junction has contributed to the inference.

\section{Splice junction representation}

A splice junction sequence is represented by four subsequences, the upstream exonic subsequence and downstream intronic subsequence at the donor site, and the 

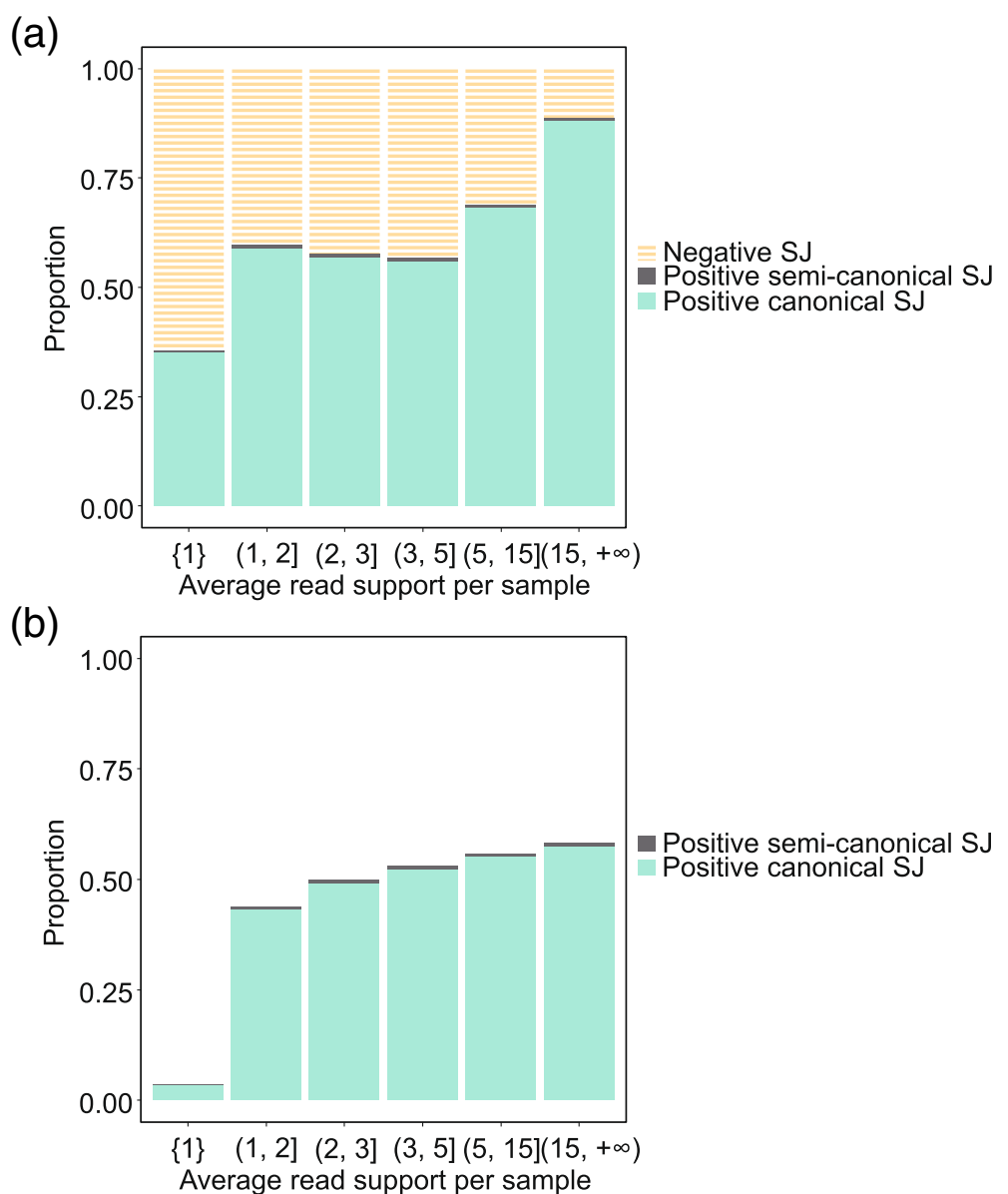

Fig. 5 Positive splice junctions tend to have high read support and contain the canonical flanking string. a Discrete proportions of negatives, positive semi-canonical splice junctions and positive canonical splice junctions from the classification results, given the average read support per sample. Splice junctions with average read support per sample more than 15 achieve a positive rate of around $88 \%$. In contrast, for splice junctions with average read support per sample no more than 1, only $36 \%$ are identified as positive. There is a significant rise in the probability to obtain a positive splice junction with the increase of the average read support per sample. Around $99 \%$ positive splice junctions contain the canonical flanking string. b Cumulative proportions of positive semi-canonical and canonical splice junctions with the increase of the average read support per sample

upstream intronic subsequence and downstream exonic subsequence at the acceptor site, as shown in Fig. 8. Each subsequence has the length of 30 , which is believed to be optimal for splice site/junction prediction $[19,22,26,27$, 48]. Nucleotides in each sequence are represented through one-hot encoding, in which $\mathrm{A}, \mathrm{C}, \mathrm{G}, \mathrm{T}$ and $\mathrm{N}$ are encoded by $[0,0,0,1][0,0,1,0,0,1,0,0][1,0,0,0]$ and $[0.25$, $0.25,0.25,0.25]$ respectively. In the proposed encoding system, the orthonormal sparse encoding is used for the four definite values (A, C, G and T) as it has been used widely in the numerical representations of biological sequences [49]. But for the ambiguous base $\mathrm{N}$, instead of disregarding it or giving it the same importance as the definite values, the probability is used.

Each splice junction sequence is transformed into a 3-dimensional tensor of shape [height, width, channels]. The first dimension 'height' is equal to one, and the second dimension 'width' indexes the sequence length, that is, the number of nucleotides in the sequence, and the third dimension 'channels' indexes the type of nucleotide. The tensors are fed as input to deep convolutional neural networks for downstream processing.

\section{Deep convolutional neural network}

DeepSplice contains a multi-layer feedforward neural network. We stack one input layer, two convolutional layers, one fully connected layer, and one output layer. The whole network architecture can be written as follows:

$$
\text { Label of class }=f_{\text {fcn }}\left(f_{\text {conv } 2}\left(f_{\text {conv } 1}(\text { Sequence nucleotide signal })\right)\right.
$$

In this way, the convolutional neural network transforms the nucleotide signal in splice junction sequences to the final label of class as shown in Fig. 8. 


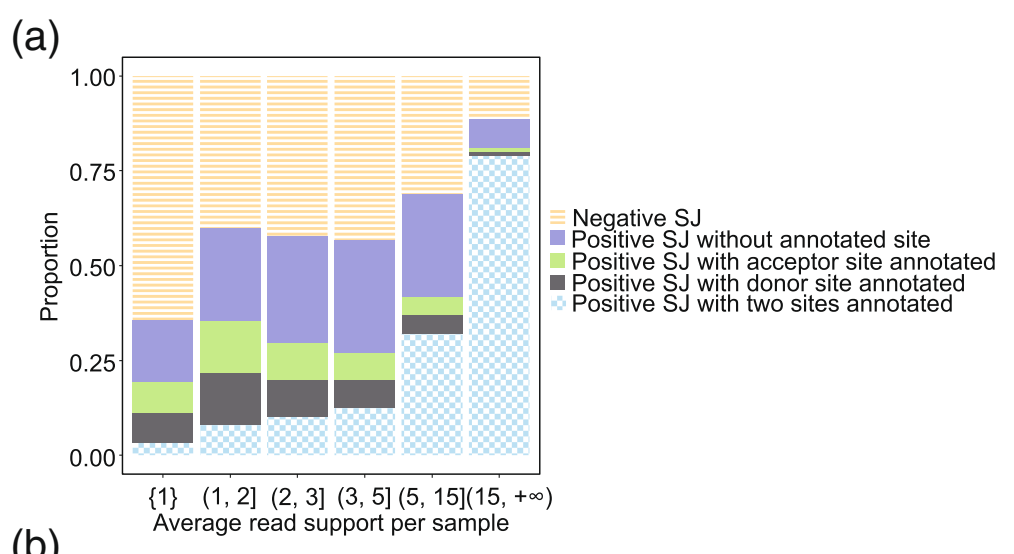

(b)

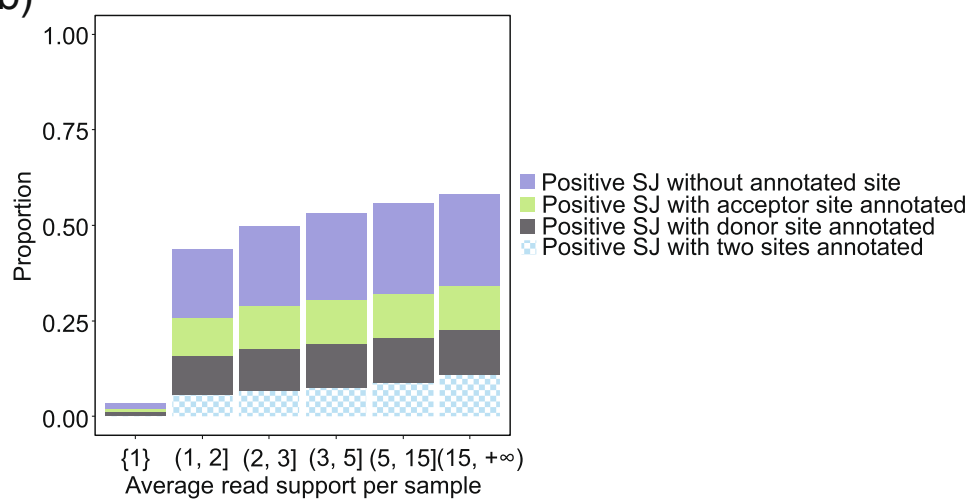

Fig. 6 Positive splice junctions tend to have both donor and acceptor sites annotated. a Discrete proportions of negatives, positive splice junctions without annotated site, positive splice junctions with acceptor site annotated, positive splice junctions with donor site annotated and positive splice junctions with two sides annotated, given the average read support per sample. 97\% of splice junctions with both sites annotated are classified as positives, while only 39\% with both sites being novel are positive. Splice junctions connecting annotated splice sites also tend to be associated with higher read coverage. $\mathbf{b}$ Cumulative proportions of positive splice junctions in each category with the increase of the average read support per sample

In the first convolutional layer, the convolution will compute 8 features over the input tensor which represents splice junction sequence, which results in 8 feature maps of the input tensor. In order to reason the complex nonlinearity between inputs and outputs, we further stack the second convolutional layer computing 16 features over 8 feature maps from the first convolutional layer. In the convolutional layers, the filters have size $3 \times 1$. During the forward pass, we slide each filter along the splice junction sequence and compute dot products between the filter and the input tensor. As we slide the filter over the input splice junction sequence we will produce feature maps that give the responses of that filter at every spatial position. After two convolutional layers, the features are presented in 16 tensors. The output of the second convolutional layer is taken by a fully connected layer with 32 feature maps for high-level reasoning. The fully connected layer is followed by the output layer indicating the final label of class. In the neural network, all parameters are learned during training to minimize a loss function which captures the difference between the true labels of class and predicted values.
Training the network follows the standard backpropagation and optimizes the loss function using Adam [50]. Advance deep learning techniques L2 regularization [51], dropout [52] and mini-batch gradient descent [53] are deployed to regularize the network to prevent over-fitting and to accelerate the training process.

In the reference step, testing splice junction sequences transformed by one-hot encoding are fed to the learned network for a binary classification, which outputs the predicted label of the class, true or false splice junction.

\section{Deep Taylor decomposition of deep convolutional neural network}

We propose to use deep Taylor decomposition [40] to explain the contribution of nucleotides in the splice junction sequence to the final decision function of the deep convolutional neural network, as shown in Fig. 9. Taking image recognition task as an example, such decomposition results in a "heat map" that indicates what pixels of the image are important for a neural network classification. In our application, for testing splice junction sequence $\mathbf{S}$, we would like to associate to nucleotide $n$ a 
Splice junction

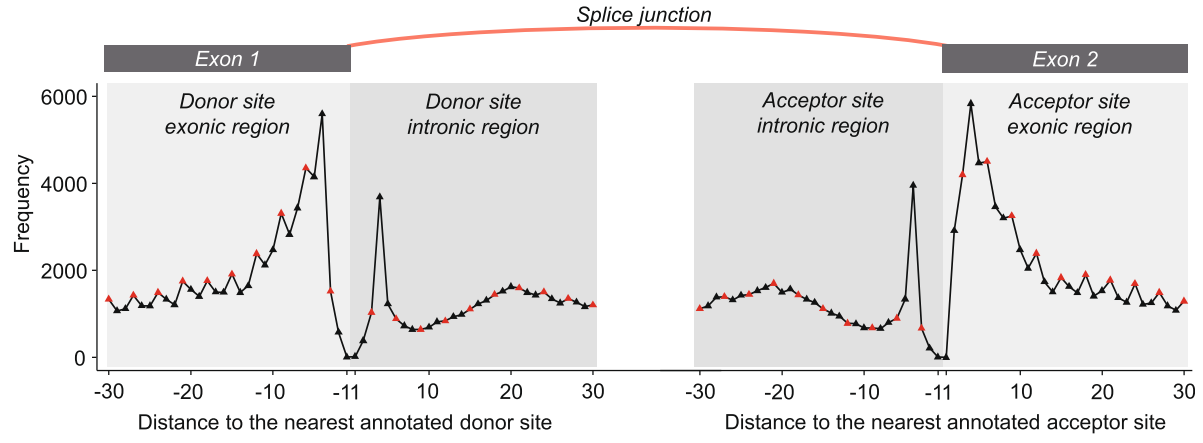

Fig. 7 Splice sites which maintain the coding frame of the exon are observed more often than those which disrupt frame. Positive splice junctions in intropolis near known protein-coding junctions show a periodic pattern. For each donor (acceptor) site in the positive splice junctions, we calculated its distance to the nearest annotated donor (acceptor) site, and then counted the frequency for each position. The red points denote positions that are a multiple of three base pairs from the major splice form, and the black points those that are not

contribution score $C_{n}(\mathbf{S})$ from which it is possible to judge which nucleotides are of importance to explain the predicted label of class from the deep convolutional neural network.

Deep Taylor decomposition operates by running a backward pass on the trained convolutional neural network using a predefined set of rules. To decompose $C_{j}$ on the set of lower layer neurons $\left\{x_{i}\right\}$ to which $x_{j}$ is connected, Taylor decomposition [54] is employed, and $C_{j}$ is given by:

$C_{j}=\left(\frac{\partial C_{j}}{\partial\left\{x_{i}\right\}} \tilde{x}_{\left.\tilde{x}_{i}\right\}^{(j)}}\right)^{\mathrm{T}} \cdot\left(\left\{x_{i}\right\}-\left\{\tilde{x}_{i}\right\}^{(j)}\right)+\varepsilon_{j}=\left.\sum_{i} \frac{\partial C_{j}}{\partial x_{i}}\right|_{\left\{\widetilde{x}_{i}\right\}^{(j)}} \cdot\left(x_{i}-\widetilde{x}_{i}^{(j)}\right)+\varepsilon_{j}=\sum_{i} C_{i j}+\varepsilon_{j}$

where $\varepsilon_{j}$ denotes the Taylor residual, and where indicates that the derivative has been evaluated at $\left\{\widetilde{x}_{i}\right\}^{(j)}$ root point $\left\{\widetilde{x}_{i}\right\}^{(j)}$. The identified term $C_{i j}$ is the redistributed contribution from neuron $x_{j}$ to neuron $x_{i}$ in the lower layers. To determine the total contribution of neuron $x_{i}$, one needs to pool contribution coming from all neurons $\left\{x_{j}\right\}$ to which the neuron $x_{i}$ contributes:

$$
C_{i}=\sum_{j} C_{i j}=\left.\sum_{j} \frac{\partial C_{j}}{\partial x_{i}}\right|_{\left\{\widetilde{x_{i}}\right\}^{(j)}} \cdot\left(x_{i}-{\widetilde{x_{i}}}^{(j)}\right)
$$

which will be central for computing explicit contribution redistribution formulas based on specific choices of root points $\left\{\widetilde{x}_{i}\right\}^{(j)}$. Backpropagating from the function output down to the input, it results in assigning a set of scores $\mathbf{C}(\mathbf{S})=\left\{C_{n}(\mathbf{S})\right\}$ to the nucleotides in the input testing

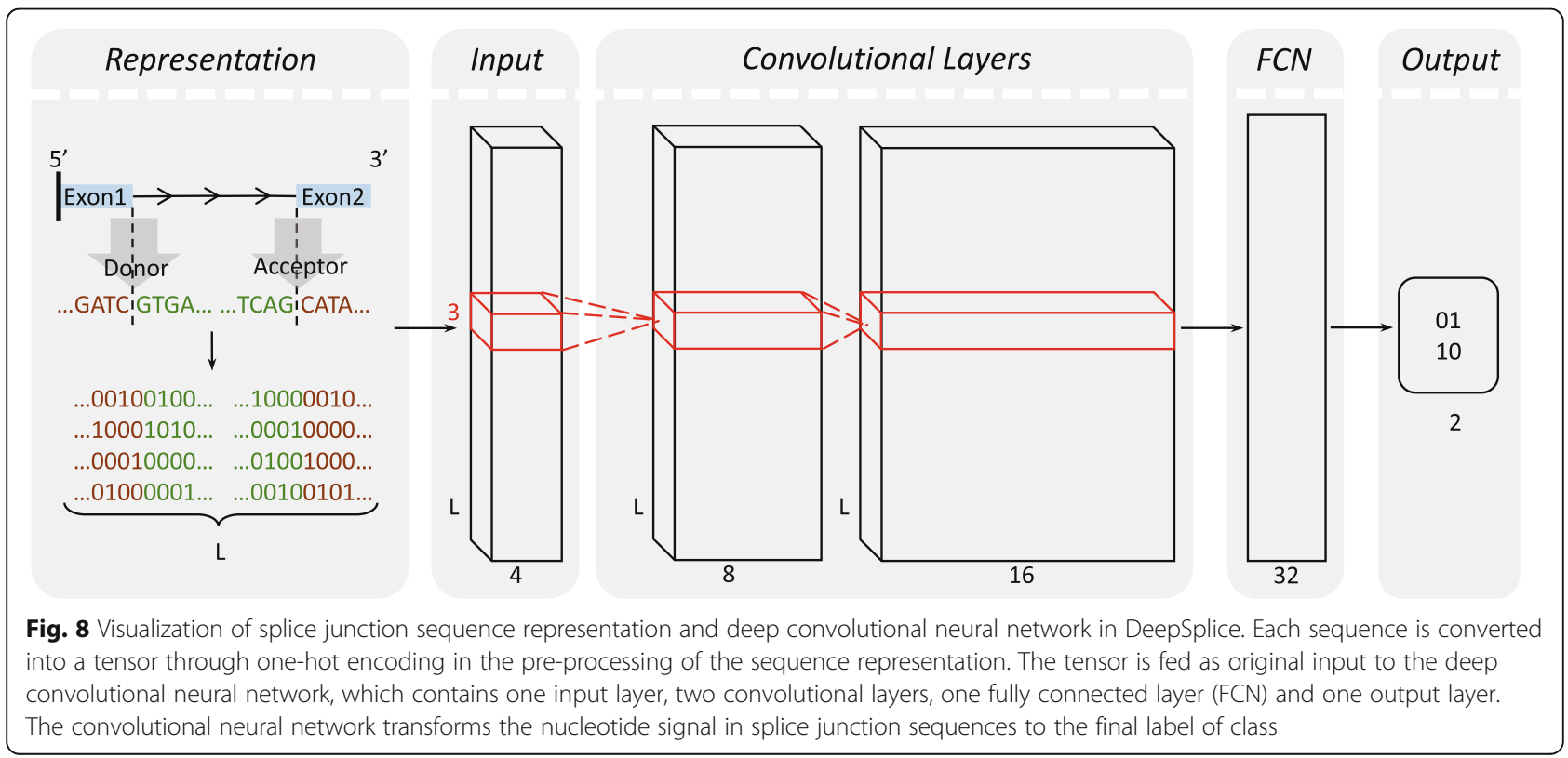




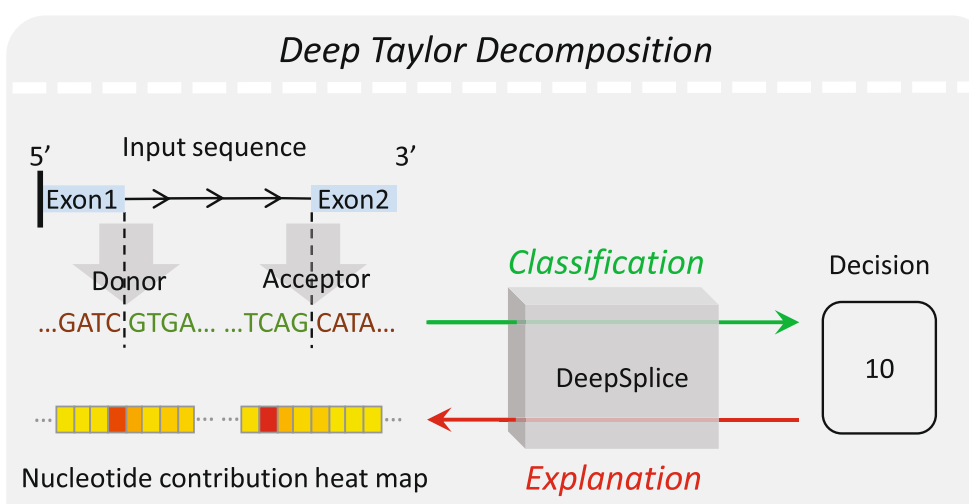

Fig. 9 Visualization of deep Taylor decomposition in DeepSplice. Deep Taylor decomposition explains the contribution of each nucleotide in the splice junction sequence to the final decision function of the deep convolutional neural network. Deep Taylor decomposition operates by running a backward pass on the trained convolutional neural network using a predefined set of rules

splice junction sequence $\mathbf{S}$ to quantify their contributions to the predicted label of class.

\section{Other deep learning architectures}

To decipher the abilities of different deep learning architectures in handling splice junction sequence data, we further build multilayer perceptron network (MLP) and long short-term memory network (LSTM) to compare with convolutional neural network. MLP is a feedforward artificial neural network with multiple hidden layers of units between input and output layers. LSTM is a recurrent neural network architecture where connections between units form a directed cycle.

The multilayer perceptron network is composed of one input layer, four hidden layers and one output layer. Each layer is fully connected to next layer in the network. The number of neurons in each hidden layer is 64 , 128, 128 and 256 respectively. In the long short-term memory network, we deploy one input layer, three hidden layers and one output layer. Each of the three hidden layers contains 16 LSTM cells. For both architectures, the inputs are splice junction sequences transformed by one-hot encoding, and the outputs are class labels. Advance deep learning techniques, dropout [52], regularization [51], mini-batch gradient descent [53] and Adam [50], are exploited in the supervised training steps in both networks.

\section{Filtering of false splice junction as a result of repetitive sequences}

One potential resource of false positive splice junction is the inability to align a sequence to the correct sites due to higher mismatches than the threshold set by aligners or small indels that cannot be detected by aligners. Before the classification of splice junctions, we first remove the splice junctions whose sequence at the acceptor (donor) site has high sequence similarity with the immediate flanking sequence next to the donor (acceptor) site or the sequence at any of its alternative acceptor (donor) sites, as shown in Fig. 10. The edit distance between the alternative acceptor (donor) site sequences is computed using the Smith-Waterman algorithm [55]. This filtering strategy is independent of read coverage and enables the retention of correct splice junctions even with low read

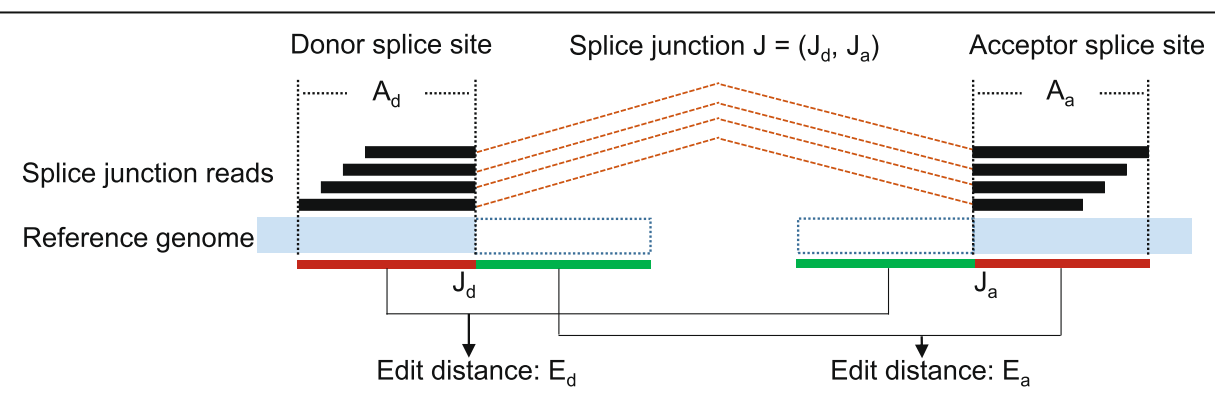

Fig. 10 Illustration of splice junction filtering strategy. In this example, two edit distances are calculated. One $\left(E_{d}\right)$ is between anchor sequence at donor site $\left(G\left[J_{d}-A_{d}+1: J_{d}\right]\right)$ and intermediate flanking sequence next to acceptor site $\left(G\left[J_{a}-A_{a}: J_{a}-1\right]\right)$. The other $\left(E_{a}\right)$ is between anchor sequence at acceptor site $\left(G\left[J_{a}: J_{a}+A_{a}-1\right]\right)$ and intermediate flanking sequence next to donor site $\left(G\left[J_{d}+1: J_{d}+A_{d}\right]\right)$ 
coverage. The removal of these sequences is necessary as most of them are highly similar with one of the splice junctions remaining in the data set.

\section{Implementation}

The deep learning architectures are implemented using TensorFlow [56]. Training and testing are deployed on Nvidia GeForce GTX 1080 graphics cards. DeepSplice is freely available for academic use and can be accessible at https://github.com/zhangyimc/DeepSplice.

\section{Performance measures}

We employ the following metrics: Area Under the ROC Curve (auROC), Area Under the Precision Recall Curve (auPRC), sensitivity, specificity, accuracy, $\mathrm{F}$ measure and $Q^{9} . Q^{9}$ is independent of the class distribution in the data set and is used to evaluate the classifier performance on splice site prediction [35], which is defined as: $Q^{9}=\left(1+q^{9}\right) /$ 2 where

$$
q^{9}=\left\{\begin{array}{c}
(T N-F P) /(T N+F P), \text { if } T P+F N=0 \\
(T P-F N) /(T P+F N), \text { if } T N+F P=0 \\
1-\sqrt{2} \sqrt{\left[F N /\left(T P+F N^{2}+[F P /(T N+F P)]^{2}\right.\right.}, \text { if } T P+F N \neq 0 \text { and } T N+F P \neq 0
\end{array} .\right.
$$

\section{Additional file}

Additional file 1: Figures S1, S2, S3, S4 and S5. Table S1 and S2. (PDF $1105 \mathrm{~kb})$

\section{Abbreviations}

SRA: Sequence Read Archive; HS3D: Homo Sapiens Splice Sites Database; CNN: convolutional neural network; ConvNet: convolutional neural network; MLP: multilayer perceptron network; LSTM: long short-term memory network; aUROC: Area Under the ROC Curve; auPRC: Area Under the Precision Recall Curve

\section{Acknowledgments}

Not applicable.

\section{Consent to publication}

Not applicable.

\section{Funding}

This work was supported by National Science Foundation [CAREER award grant number 1054631 to J.L.]; and National Institutes of Health [grant number P30CA177558 and 5R01HG006272-03 to J.L.]. The funding body had no role in the design of the study and collection, analysis, and interpretation of data and in writing the manuscript.

\section{Availability of data and materials}

The dataset supporting the conclusions of this article is available in the GitHub repository, https://github.com/zhangyimc/DeepSplice.

\section{Authors' contributions}

Y.Z., J.L. and X.L. conceived and developed the research idea. Y.Z. performed the software implementation and data collection. Y.Z. performed the computational experiments with the assistance of J.L. and X.L. The original draft was written by Y. Z with technical content from X.L. and extensive review and editorial input from J.L. and J.N.M. All authors read and approved the final manuscript.

\section{Ethics approval and consent to participate}

No permission was required from the ethics committee as the project did not involve testing of human, animal or endangered plant species subjects.

\section{Competing interests}

The authors declare that they have no competing interests.

\section{Publisher's Note}

Springer Nature remains neutral with regard to jurisdictional claims in published maps and institutional affiliations.

\section{Author details}

'Department of Computer Science, University of Kentucky, Lexington, KY 40506, USA. ${ }^{2}$ Department of Veterinary Science, University of Kentucky, Lexington, KY 40506, USA.

Received: 16 November 2017 Accepted: 3 December 2018

Published online: 27 December 2018

\section{References}

1. Wang $E T$, et al. Alternative isoform regulation in human tissue transcriptomes. Nature. 2008;456(7221):470-6.

2. Roy B, Haupt LM, Griffiths LR. Review: alternative splicing (AS) of genes as an approach for generating protein complexity. Curr Genomics. 2013;14(3): 182-94.

3. Cloonan $\mathrm{N}$, et al. Stem cell transcriptome profiling via massive-scale mRNA sequencing. Nat Methods. 2008;5(7):613-9.

4. Marioni JC, et al. RNA-seq: an assessment of technical reproducibility and comparison with gene expression arrays. Genome Res. 2008;18(9):1509-17.

5. Mortazavi A, et al. Mapping and quantifying mammalian transcriptomes by RNA-Seq. Nat Methods. 2008;5(7):621-8.

6. Sultan $\mathrm{M}$, et al. A global view of gene activity and alternative splicing by deep sequencing of the human transcriptome. Science. 2008;321(5891):956-60.

7. Dobin A, et al. STAR: ultrafast universal RNA-seq aligner. Bioinformatics. 2013;29(1):15-21.

8. Kim D, Langmead B, Salzberg SL. HISAT: a fast spliced aligner with low memory requirements. Nat Methods. 2015;12(4):357-60.

9. Kim D, et al. TopHat2: accurate alignment of transcriptomes in the presence of insertions, deletions and gene fusions. Genome Biol. 2013;14(4):R36

10. Wang $K$, et al. MapSplice: accurate mapping of RNA-seq reads for splice junction discovery. Nucleic Acids Res. 2010. https://doi.org/10.1093/nar/gkq622

11. Wu TD, Nacu S. Fast and SNP-tolerant detection of complex variants and splicing in short reads. Bioinformatics. 2010;26(7):873-81.

12. Libby $\mathrm{P}$, Bonow RO, Mann DL, et al. Braunwald's heart disease: a textbook of cardiovascular medicine. Philadelphia: WB Saunders Co.; 2007. p. p579.

13. Li Y, et al. TrueSight: a new algorithm for splice junction detection using RNA-seq. Nucleic Acids Res. 2013;41(4):e51.

14. Nellore A, et al. Human splicing diversity and the extent of unannotated splice junctions across human RNA-seq samples on the sequence read archive. Genome Biol. 2016;17(1):266.

15. Nellore A, et al. Rail-RNA: Scalable analysis of RNA-seq splicing and coverage. Bioinformatics. 2016;33(24):4033-40.

16. Hu Y, et al. DiffSplice: the genome-wide detection of differential splicing events with RNA-seq. Nucleic Acids Res. 2012;41(2):e39.

17. Trapnell $\mathrm{C}$, et al. Differential gene and transcript expression analysis of RNAseq experiments with TopHat and cufflinks. Nat Protoc. 2012;7(3):562-78.

18. Gatto A, et al. FineSplice, enhanced splice junction detection and quantification: a novel pipeline based on the assessment of diverse RNA-Seq alignment solutions. Nucleic Acids Res. 2014;42(8):e71.

19. Pickrell JK, et al. Noisy splicing drives mRNA isoform diversity in human cells. PLoS Genet. 2010:6(12):e1001236.

20. Stormo GD, et al. Use of the 'Perceptron'algorithm to distinguish translational initiation sites in E. coli. Nucleic Acids Res. 1982;10(9): 2997-3011.

21. Noordewier MO, Towell GG, Shavlik JW. Training knowledge-based neural networks to recognize genes in DNA sequences. Adv Neural Inf Proces Syst. 1991;3:530-6.

22. Brunak S, Engelbrecht J, Knudsen S. Prediction of human mRNA donor and acceptor sites from the DNA sequence. J Mol Biol. 1991;220(1):49-65. 
23. Degroeve $S$, et al. SpliceMachine: predicting splice sites from highdimensional local context representations. Bioinformatics. 2005;21(8): 1332-8.

24. Huang J, et al. An approach of encoding for prediction of splice sites using SVM. Biochimie. 2006:88(7):923-9.

25. Sonnenburg $\mathrm{S}$, et al. Accurate splice site prediction using support vector machines. BMC Bioinforma. 2007:8(10):S7.

26. Reese MG, et al. Improved splice site detection in genie. J Comput Biol. 1997:4(3):311-23.

27. Pertea M, Lin X, Salzberg SL. GeneSplicer: a new computational method for splice site prediction. Nucleic Acids Res. 2001;29(5):1185-90.

28. Baten AK, et al. Splice site identification using probabilistic parameters and SVM classification. BMC Bioinforma. 2006;7(5):S15.

29. Lee T, Yoon S. Boosted Categorical Restricted Boltzmann Machine for Computational Prediction of Splice Junctions. In: International Conference on Machine Learning, France, 2015. p. 2483-92.

30. Chuang, J.S. and D. Roth. Splice Site Prediction Using a Sparse Network of Winnows. 2001, University of Illinois at Urbana-Champaign.

31. Zhang MQ. Identification of protein coding regions in the human genome by quadratic discriminant analysis. Proc Natl Acad Sci. 1997;94(2):565-8.

32. Zhang $Y$, et al. Splice site prediction using support vector machines with a Bayes kernel. Expert Syst Appl. 2006;30(1):73-81.

33. Wei $D$, et al. A novel splice site prediction method using support vector machine. J Comput Inform Syst. 2013;920:8053-60.

34. Yeo $G$, Burge CB. Maximum entropy modeling of short sequence motifs with applications to RNA splicing signals. J Comput Biol. 2004;11(2-3):377-94.

35. Zhang Q, et al. Splice sites prediction of human genome using length-variable Markov model and feature selection. Expert Syst Appl. 2010;37(4):2771-82.

36. Ghandi $M$, et al. Enhanced regulatory sequence prediction using gapped kmer features. PLoS Comput Biol. 2014;10(7):e1003711.

37. Harrow J, et al. GENCODE: the reference human genome annotation for the ENCODE project. Genome Res. 2012;22(9):1760-74.

38. Li J, et al. High-accuracy splice site prediction based on sequence component and position features. Genet Mol Res. 2012;11(3):3432-51.

39. Pollastro P, Rampone S. HS3D, a dataset of Homo Sapiens splice regions, and its extraction procedure from a major public database. International Journal of Modern Physics C. 2002;13(08):1105-17.

40. Montavon G, et al. Explaining nonlinear classification decisions with deep Taylor decomposition. Pattern Recogn. 2017;65:211-22.

41. Sercu T, et al. Very deep multilingual convolutional neural networks for LVCSR. In: 2016 IEEE International Conference on Acoustics, Speech and Signal Processing (ICASSP), China, 2016;4955-9.

42. Zhang Y, et al. DeepSplice: Deep classification of novel splice junctions revealed by RNA-seq. In: 2016 IEEE International Conference on Bioinformatics and Biomedicine (BIBM), China, 2016. p. 330-3.

43. Hogg RV, Craig AT. Introduction to mathematical statistics. (5"' edition). Upper Saddle River, New Jersey: Prentice Hall; 1995.

44. Burset M, Seledtsov I, Solovyev V. Analysis of canonical and noncanonical splice sites in mammalian genomes. Nucleic Acids Res. 2000; 28(21):4364-75.

45. Leung MK, et al. Deep learning of the tissue-regulated splicing code. Bioinformatics. 2014;30(12):i121-9.

46. Xiong HY, et al. The human splicing code reveals new insights into the genetic determinants of disease. Science. 2015;347(6218):1254806.

47. Sibley CR, Blazquez $L$, Ule J. Lessons from non-canonical splicing. Nat Rev Genet. 2016;17(7):407-21.

48. Senapathy P, Shapiro MB, Harris NL. [16] Splice junctions, branch point sites, and exons: Sequence statistics, identification, and applications to genome project. Methods Enzymol. 1990;183:252-78.

49. Rampone S. Recognition of splice junctions on DNA sequences by BRAIN learning algorithm. Bioinformatics. 1998;14(8):676-84.

50. Kingma D and Ba J. Adam: A method for stochastic optimization. arXiv preprint arXiv:1412.6980, 2014.

51. Ng, A.Y. Feature selection, L 1 vs. L 2 regularization, and rotational invariance. In: Proceedings of the twenty-first international conference on Machine learning, New York, 2004. p. 78-85.

52. Srivastava N, et al. Dropout: a simple way to prevent neural networks from overfitting. J Mach Learn Res. 2014;15(1):1929-58.

53. Konečný J, et al. Mini-batch semi-stochastic gradient descent in the proximal setting. IEEE J Sel Top Sig Process. 2016;10(2):242-55.
54. Kanwal R, Liu K. A Taylor expansion approach for solving integral equations. Int J Math Educ Sci Technol. 1989;20(3):411-4.

55. Smith TF, Waterman MS. Identification of common molecular subsequences. J Mol Biol. 1981;147(1):195-7.

56. Abadi M, et al. Tensorflow: a system for large-scale machine learning. In: OSDI, USA. 2016;16:265-83.
Ready to submit your research? Choose BMC and benefit from:

- fast, convenient online submission

- thorough peer review by experienced researchers in your field

- rapid publication on acceptance

- support for research data, including large and complex data types

- gold Open Access which fosters wider collaboration and increased citations

- maximum visibility for your research: over $100 \mathrm{M}$ website views per year

At BMC, research is always in progress.

Learn more biomedcentral.com/submissions 